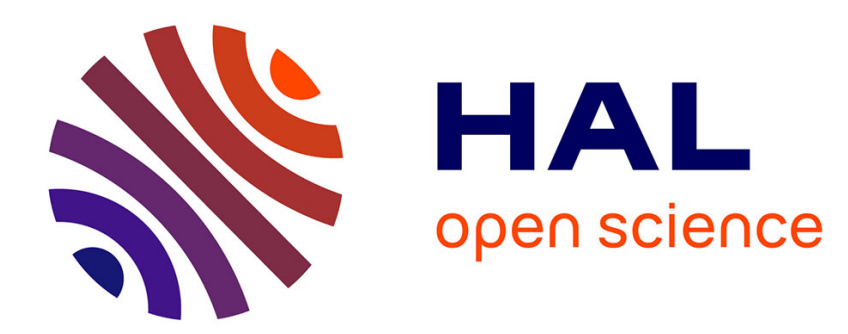

\title{
Solar irradiation nowcasting by stochastic persistence: a new parsimonious, simple and efficient forecasting tool
}

\author{
Cyril Voyant, Gilles Notton
}

\section{To cite this version:}

Cyril Voyant, Gilles Notton. Solar irradiation nowcasting by stochastic persistence: a new parsimonious, simple and efficient forecasting tool. Renewable and Sustainable Energy Reviews, 2018, 92, pp.343-352. hal-01783937

\section{HAL Id: hal-01783937 \\ https://hal.science/hal-01783937}

Submitted on 2 May 2018

HAL is a multi-disciplinary open access archive for the deposit and dissemination of scientific research documents, whether they are published or not. The documents may come from teaching and research institutions in France or abroad, or from public or private research centers.
L'archive ouverte pluridisciplinaire HAL, est destinée au dépôt et à la diffusion de documents scientifiques de niveau recherche, publiés ou non, émanant des établissements d'enseignement et de recherche français ou étrangers, des laboratoires publics ou privés. 


\section{Solar irradiation nowcasting by stochastic persistence: a new parsimonious, simple and efficient} forecasting tool

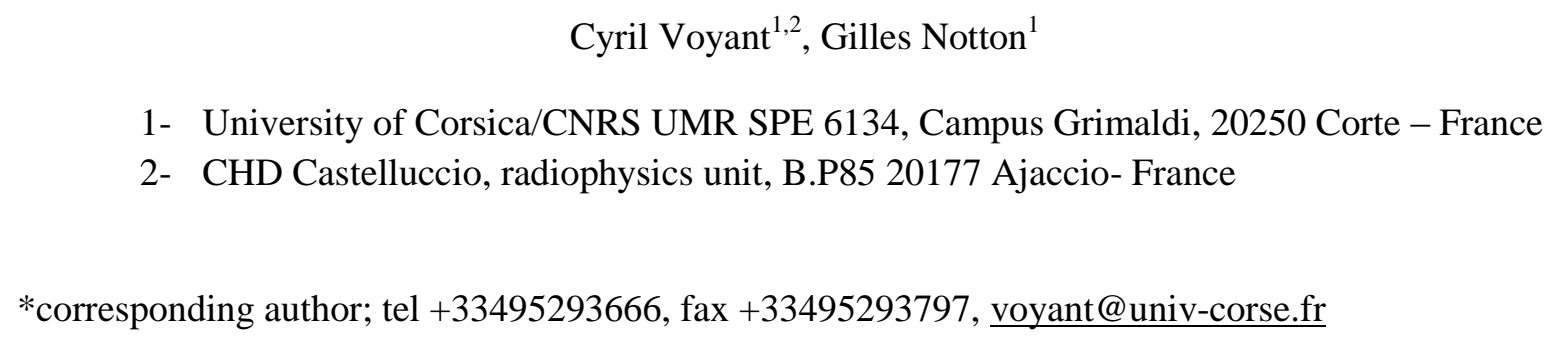

\section{Abstract}

Simple, naïve, smart or clearness persistences are tools largely used as naïve predictors for the global solar irradiation forecasting. It is essential to compare the performances of sophisticated prediction approaches with that of a reference approach generally a naïve methods. In this paper, a new kind of naïve "nowcaster" is developed, a persistence model based on the stochastic aspect of measured solar energy signal denoted stochastic persistence and constructed without needing a large collection of historical data. Two versions are proposed: one based on an additive and one on a multiplicative scheme; a theoretical description and an experimental validation based on measurements realized in Ajaccio (France) and Tilos (Greece) are exposed. The results show that this approach is efficient, easy to implement and does not need historical data as the machine learning methods usually employed. This new solar irradiation predictor could become an interesting tool and become a new member of the solar forecasting family. 


\section{Introduction}

\subsection{Interest of solar irradiation forecasting}

Over the last ten years, energy market was boosted with the advent of renewable energies and in particular thanks to solar energy. The main interest of this kind of primary energy is to be easily and cleanly transformed into electricity particularly via photovoltaic conversion [1], which is the most flexible form of energy [2]. The main problem concerning the use of solar energy is its continuous variability relating both to time and space $[3,4]$. The variability can be divided into two components, the first one denoted deterministic part and the second one stochastic or random part. If the deterministic component is generated by the movements of rotation and revolution of the Earth [5], the stochastic component is generated by weather and cloud occurrences [6]. Solar energy intermittency has a great influence on the output power of photovoltaic $(P V)$ plants, which can fluctuate significantly in short intervals (related to the random part) and in long intervals (related to daily and yearly seasonal effects) [7]. This no-controllable intermittence has negative consequences on the management of the electrical distribution and stability (forcing to limit the penetration rate of such intermittent energy systems) and on the $\mathrm{kWh}$ production costs [8]. One way to solve or to reduce this problem is to forecast this PV output power [9]. A good forecast helps the grid manager to plan the other energy capabilities to compensate for the $P V$ plants power variations [10]. The forecasting quality of the ouput PV plant is strongly linked to the global horizontal irradiation (GHI) forecasting accuracy [11]. Some authors go even further and consider the problem of $P V$ output power forecasting and the solar irradiance forecasting problem as equal [12]. In this paper, a new forecasting tool is developed and tested in view to assist the electrical grid manager by predicting easily GHI.

\subsection{Prediction and Parsimony}

Time series forecasting [13] consists to estimate possible events or their evolutions by using as tools the past and the present. Before exposing the deferent tools available in order to nowcast $G H I$, it is important to define the "time series" term and the word "prediction" related to this kind of mathematical tools $[14,15]$.

Definition 1.1. Time series: A univariate time series is a sequence of measurements of the same variable collected over time. Most often, the measurements are made at regular time intervals. The common notation concerning a time series of $G H I$ measurement is $G H I=\{G H I(t): t \in T\}$ where $T$ is the index set. 
Remark 1.1. GHI (nondeterministic) time series may be analyzed by assuming they are partly the manifestations of stochastic (random) processes [16-18] which is a statistical phenomenon consisting of a collection of random variables ordered in time and evolving according to a priori unknown probabilistic laws.

To succeed a time series prediction, only four conditions must be fulfilled:

- a certain regularity in the functioning of the studied process,

- this regularity must provide information on the future,

- the method chosen to establish the prediction captures a part of this regularity,

- the prediction will be efficient if and only if the "noise" or past irregularities are excluded as far as possible.

Forecasting the solar irradiation from 1 hour to 6 hours (defining the nowcasting [12]) is currently done using statistical or machine learning methods coupled to time series analysis. Many papers show that these methods yield similar results [19-21], none appears to outperform other and sometimes simple methods propose very similar results. According to a review analysis [22], it seems that it is not interesting to predict with very complex methods because a gain of tenths of a percent on the forecasting performances has only a small (but not negligible) impact on the grid management. Moreover, in [8], authors model a fictive solar plant with a nominal capacity of $1000 \mathrm{~kW}$ and show that a large nRMSE reduction from $32 \%$ to $28 \%$ (-4 percentage points) allows a financial saving close to $9 \%$, so $70 €$ per day for the considered installation. In fact, the electricity grid operator needs a reliable tool which is adaptable for all horizons (between 5 minutes and 6 hours). The ideal case is to elaborate a tool which does not require a large learning history [15] in order to be quickly deployed on any site. In this paper we propose a new very simple and parsimonious tool based on the persistence of stochastic signal. Note that if in the operational case, the prediction with persistence does not need a large historical data (only a few hours), the present study is a retrospective comparison and is operated with historical data. The idea behind parsimonious models stems from the 14th century and the formulation of the Occam's razor [23] stating that "we should use no more parameters than necessary to explain the model well. There is generally a tradeoff between goodness of fit and parsimony. Models with many parameters (as machine learning tools [24,25]) tend to have a better fit than high parsimony models (as persistence), however this is not usually a good thing. Indeed, adding more parameters usually results in a good model fit for the data at hand, but that same model will likely be useless for predicting other data sets. In [26] (pp. 103-104), sentences summarize the interpretation related to simple models results: «Sometimes a simple model will outperform a more complex model . . . Nevertheless, I believe that deliberately limiting the complexity of the model is not fruitful when the problem is evidently complex. Instead, if a simple model is found that outperforms some particular complex model, the appropriate response is to define a different complex model that captures whatever 
aspect of the problem led to the simple model performing well». It is essential to correctly study the simple models before to elaborate more sophistical approaches. Reference models should be well chosen to truly and objectively decide on the quality of the forecast.

\section{Machine learning or simple models of persistence}

Machine learning [27] is a branch of artificial intelligence [28]. It concerns the construction and the study of systems that can learn from data sets, giving to computers the ability to learn without being explicitly programmed.

\subsection{Models definitions}

With the machine learning tools based predictions, the system is built from a random output (denoted variable $y$ ) and a set of random input (denoted variables $x=\left\{x_{1} \ldots, x_{n}\right\}$ ). Using a learning sample $\left\{y_{i}, x_{i}\right\}_{1}^{N}$ of known values of pairs $(y, x)$, the aim is to obtain and estimate a model function $f^{*}(\mathrm{x})$, among all the functions $f(x)$ available and which allows to map (as well as possible!) $x$ to $y$. The objective is reached after an optimization of the expected value $(\mathbb{E})$ of some specified loss functions $L(y, f(x))$ over the joint distribution of all $(y, x)$ pairs:

$f^{*}(x)=\operatorname{argmin}_{f}(\mathbb{E}(L(y, f(x)))$

Equation 1

In a regression problem, the loss function $L(y, f(x))$ includes usually 2-norm or 1-norm distances respectively computed from the squared-error $(y-f(x))^{2}$ (Euclidean norm giving more importance to large deviations or outliers) and the absolute error $|y-f(x)|$ (absolute-value norm giving importance to the trend gap). Typically in the supervised cases, the machine learning methods are confronted to bias-variance tradeoff and are very user dependent and difficult to make a good use [29]. Is machine learning is overhyped? This question was recently asked in [30], it may be time to consider other methods of modeling. The simplest method of forecasting the weather, persistence, relies upon today's conditions to forecast the conditions tomorrow. This can be a valid way of forecasting the weather when it is in a steady state, such as during the summer season when clouds are rare. This method of forecasting strongly depends upon the presence of a stagnant weather pattern. Therefore, with a fluctuating weather pattern, this method of forecasting becomes inaccurate. It can be useful in both short range forecasts and long range forecasts. The time series of global horizontal irradiation $(G H I)$ is composed by a stochastic part (Cf previous section); often when a machine learning method is used, a strong condition is necessary: the stationarity of the input data [31]. That means that the joint 
distribution of $G H I(t)$ and $G H I(t+h)$ does not depend on $t$ but only on $\mathrm{h}\left(t, h \in \mathbb{N}^{*}\right)$. To our knowledge, it is not proved that the tools used to make the GHI time series stationary allows to correctly respect this condition [32]. It is legitimate to ask: can we really use these methods even if the results are consistent ? we have of course not the answer and we would be very embarrassed to answer "no" to this question given that we ourselves abundantly study the forecast of GHI via the data driven, machine learning, artificial intelligence and others statistical methods. What is sure is that with the persistence there are both advantages: directly usable (without learning and without need of historical data) and any hypotheses or conditions concerning the model building. The "classical" persistence is not really adapted to the forecast [29] while the smart persistence (integrating a knowledge-based model using a clear sky model taking into account the sun position and the average conditions of sky state) allows to greatly improve the prediction [19].

Definition 2.1. Simple persistence: the term persistence (or simple persistence) in time series context is related to the notion of memory properties of time series, the model is built for the horizon (lookahead time) $h$ as $G H I(t+h)=G H I(t)+\varepsilon_{t+h}$, where $t$ is a time index and $\varepsilon$ denotes the residual. The forecast $\widehat{G H I}$ obtained with this model is $\widehat{G H} I(t+h)=G H I(t)$, which states that the expected value at horizon $h$ is equal to the most recent measured value.

Definition 2.2. Smart persistence: This model is based on the same assumption than persistence model but is corrected for the deterministic diurnal variation in solar irradiance, using a knowledge-based model $K(t, h): \widehat{G H} I(t+h)=G H I(t) \cdot K(t, h)$

\subsection{A short literature review on persistence}

Numerous studies show the efficiency of these naïve predictions: the persistence. In [33] the persistence is extremely detailed and authors wrote «It has been found that for short time horizons, beating persistence models is a difficult task » and demonstrated that, often, the persistence is the best method to use for the short-casting $(<1 \mathrm{~h})$ and the now-casting (1h-6h). In several studies, the simple persistence allows obtaining very good results [34- for which the difference in term of prediction error, compared with machine learning method is lower than 2.5\% [35] and in [36] lower than 5\%. Concerning the comparison between machine learning and smart (or clearness) persistence, this difference is even lower, [19] and reach $2 \%$ and the authors wrote "for hour ahead solar forecasting, the picture is less clear and seems to depend on the sky conditions“. For stable clear sky conditions (clear skies for instance), the nonlinear methods slightly improve the scaled-persistence. For unstable sky conditions, the discrepancy between the machine learning methods and the simple models is more pronounced with a $2 \% n R M S E$ difference in average. In [37] and [38] authors showed that the smart persistence is a good predictor compared to more complicated methods with an increasing of $n R M S E$ of $1 \%$. In [39] and [40] the persistence is sometime as efficient as sophisticated models while in [35] it 
is better than support vector machine. Note than the smart persistence use depends on the clear sky model use as described in [41]. In conclusion, it appears that the persistence should be an interesting forecasting. However, keep in mind that the atmospheric dynamics has major importance, and cannot be dismissed from the predictors without affecting their performance, especially when the prediction time horizon is larger than 1 hour. So, in theory, this kind of prediction based on the persistence of the phenomenon is dedicated to the very short horizons and will never be as powerful as models based on atmospheric dynamics.

\section{Stochastic persistence formalisms}

As all techniques for estimating derivatives of a noisy signal, persistence suffers from a high sensitivity to noise (or quick fluctuations). To quantify the noise related to a time series, it is common to estimate the Signal Noise Ratio (SNR) defined by the ratio between the average of the signal and the noise (standard deviation of the time series). It is a multiplicative inverse of the variation coefficient [42]. On one year and for an hourly time granularity in Tilos (Greece, 1 hour horizon), SNR varies between 0.8 and 0.6 respectively in summer and winter. When this parameter is high the persistence or smart persistence gives very good results (in summer $n R M S E=8.7 \%$ for smart persistence) but becomes less interesting and efficient when SNR decreases (in winter $n R M S E=17.4 \%$ for smart persistence). In this paper, we propose to modify the persistence estimation considering the fact that the studied series are noisy time series and thus the stochastic aspect of the measured signal will be taken into account. Note that, as $S N R$ varies, the variability varies also, so it is very complicated for a machine learning method to take into account all these characteristics without considered additional informations such as exogenous data or dummy temporal variables. The simple persistence is described in the definition 2.1, for the definition of the smart persistence, the function $K(t, h)$ (in the definition 2.2) is usually defined as the ratio $C S(t)$ of the solar radiation at the ground level on the estimated clear sky solar radiation $(C S(t)$ is computed using the well-known Solis model $[43,44])$ as described by :

$\widehat{G H} I(t+h)=G H I(t) \cdot \frac{C S(t+h)}{C S(t)}$

Equation 2

In fact, this reference predictor is built from the persistence of the clear sky index $(\operatorname{CSI}(t)=$ $G H I(t) / C S(t))$ and thus:

$\widehat{\operatorname{CSI}}(t+h)=\operatorname{CSI}(t)$

Equation 3 
We notice that one naturally transform the ratio to trend to an additive model by passing to the $\log (\mathrm{Cf}$ Box-Cox transformation, logarithmic transformation is often necessary to stabilize the variance) with $\log (\widehat{C S I}(t+h))=\log (\operatorname{CSI}(t))$, hence although it is never used in solar irradiation forecasting, another definition of the smart persistence could be:

$\log (\widehat{G H} I(t+h))=\log (G H I(t))+\log (C S(t+h))-\log (C S(t))$

Equation 4

As we will see later, the inclusion of atmospheric variables $(C S)$ into the prediction process for solar radiation will improve its performance. Two definitions (arithmetic and geometric means) are necessary to understand the next sections, either a $G H I$ time series defined by $\{G H I(t): t \in T\}$ with $\max (T)>N$ and $N \in \mathbb{N}^{*}$ :

Definition 3.1. Arithmetic mean at time $t$ for the series $x$ denoted $\overline{x_{N}}(t)=\frac{1}{N} \sum_{i=0}^{N-1} x(t-i)$

Definition 3.2. Geometric mean at time $t$ for the series $x$ denoted ${\overline{x_{N}}}^{G}(t)=\left(\prod_{i=0}^{N-1} x(t-i)\right)^{1 / N}$

\subsection{Additive scheme of the stochastic persistence}

It is possible to define a time series as a sum of 2 other series referring to Wold's theorem (or also to Cartier Perrin theorem) $[45,46]$. These theorems say that every covariance-stationary time series can be written as the sum of two time series, one deterministic and one stochastic; in our case, we can write : $G H I(t)=T(t)+\sum_{j=0}^{\infty} b_{j} \mathrm{E}(t-j)$ with $\mathrm{E}(t)$ is an uncorrelated sequence which is the innovation process (or white noise) that is the input to the linear filter $\left\{b_{j}\right\}$. $\mathrm{b}$ is the possibly infinite vector of moving average weights and $T(t)$ is a deterministic time series. Note that the stochastic part defines a new time series $\varepsilon(\mathrm{t})=\sum_{\mathbf{j}=0}^{\infty} b_{\mathrm{j}} \mathrm{E}(\mathrm{t}-\mathrm{j})$ thus in the following, we consider the decomposition form $\operatorname{GHI}(\mathrm{t})=T(\mathrm{t})+\varepsilon(\mathrm{t})$. Theoretically, the $\varepsilon(\mathrm{t})$ part is not a predictable quantity, all the available prediction tools focus on the $T(\mathrm{t})$ estimation; so concerning the persistence, it seems illogical to apply and propagate a random term to the future. In this paper, we propose new definitions of the persistence; the first one (Fig 1) is based on a model of knowledge (clear sky model; $C S$ ) and on the arithmetic mean of the difference between CS and the past GHI measurements $\Delta(t) \quad(=C S(t)-$ $G H I(t))$.

$\widehat{G H I}(t+h)=C S(t+h)-\overline{\Delta_{N}}(t)$ with $\overline{\Delta_{N}}(t)=\frac{1}{N} \sum_{i=0}^{N-1}(C S(t-i)-G H I(t-i)) \quad$ Equation 5

This averaging allows minimizing the stochastic part of the measurement, it is a low pass filter operated by moving average. From Eq (6) it comes $\left(\overline{G H I}_{N}(t)\right.$ and $\overline{C S}_{N}(t)$ arithmetic means of the $G H I$, and $C S$ ):

$\widehat{G H I}(t+h)=\overline{G H}_{N}(t)+C S(t+h)-\overline{C S}_{N}(t)$

Equation 6 
$221 \hat{\Delta}(t+h)=\bar{\Delta}_{N}(t)$

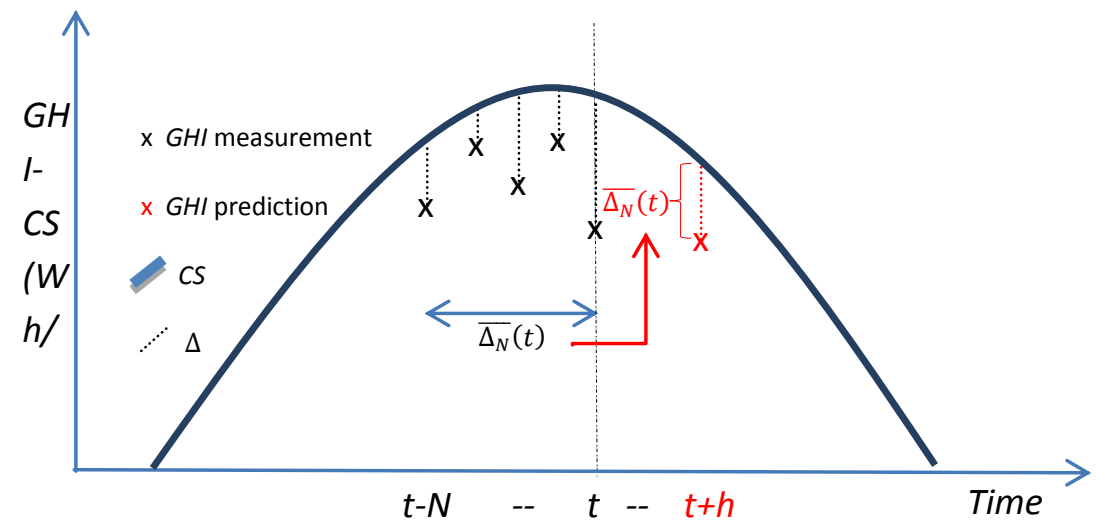

236

\subsection{Multiplicative scheme of the stochastic persistence}

238 Based on the previous subsection, we define now the persistence as a multiplicative scheme (Fig 2) 239 where:

$240 \widehat{G H} I(t+h)=C S(t+h) \cdot \overline{C S I}_{N}^{G}(t)$

$241 \overline{\operatorname{CSI}}_{N}{ }^{G}(t)$ is the geometric mean of the ratio to trend denoted clear sky index $\operatorname{CSI}(\mathrm{t})\left(=\frac{\mathrm{GHI}(\mathrm{t})}{\operatorname{CS}(\mathrm{t})}\right)$ and 242 computed with:

Thus, it comes: 


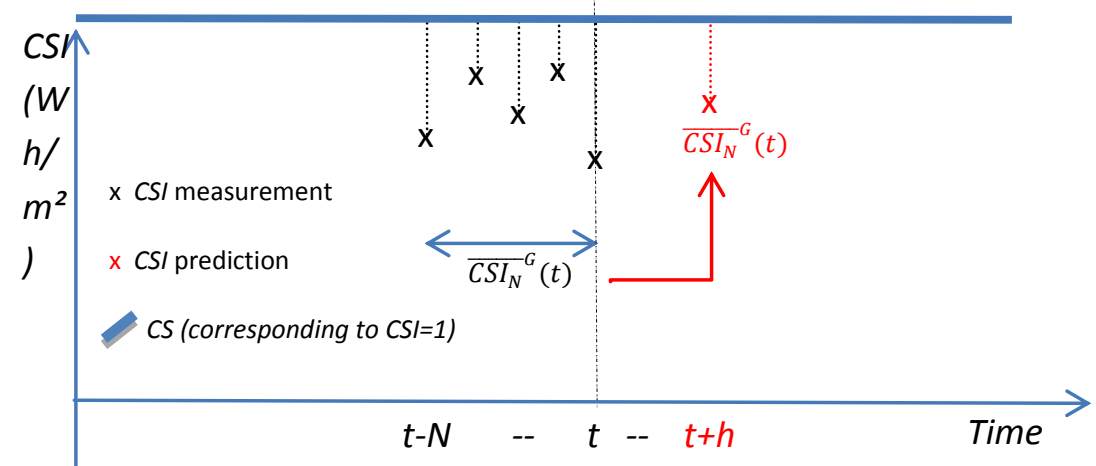

256

A similar equation (equation 11) to the smart persistence case is obtained. It is a generalized formula of the CSI persistence minimizing the random part of the measurement. If $N=1$ the equation is equivalent to the classical smart persistence equation. The reduced form is:

$\widehat{C S I}(t+h)=\overline{C S I}_{N}{ }^{G}(t)$

Equation 11

\subsection{Stochastic persistence optimization}

In Table 1 are summarized all the models defined in the previous sections.

\section{Definitions}

\begin{tabular}{|c|c|}
\hline Simple persistence $(\mathbf{P})$ & $\widehat{G H} I(t+h)=G H I(t)$ \\
\hline Smart persistence (SP) & $\begin{array}{c}\widehat{C S I}(t+h)=C S I(t) \\
\text { or } \widehat{G H I}(t+h)=G H I(t) \cdot \frac{C S(t+h)}{C S(t)}\end{array}$ \\
\hline $\begin{array}{l}\text { Stochastic persistence : } \\
\text { additive mode }\left(\text { StP }^{+}\right)\end{array}$ & $\begin{array}{c}\widehat{\Delta}(t+h)=\bar{\Delta}_{N}(t) \\
\text { or } \widehat{G H I}(t+h)=\overline{G H I}_{N}(t)+C S(t+h)-\overline{C S}_{N}(t)\end{array}$ \\
\hline $\begin{array}{c}\text { Stochastic persistence : } \\
\text { multiplicative mode }\left(S t P^{x}\right)\end{array}$ & $\begin{array}{c}\widehat{C S I}(t+h)={\overline{C S I_{N}}}^{G}(t) \\
\text { or } \widehat{G H} I(t+h)={\overline{G H I_{N}}}^{G}(t) \cdot \frac{C S(t+h)}{{\overline{C S_{N}}}^{G}(t)}\end{array}$ \\
\hline
\end{tabular}

Table 1. Short summary concerning the persistence models. 
In the additive and multiplicative cases, the optimal solution consists in quantifying $N$ which minimizes the prediction error (mean square error; $M S E$ ), then:

$$
\operatorname{argmin}\left\{\operatorname{MSE}\left(\widehat{G H I}(t+h)-\operatorname{GHI}(t+h) \mid N \in \mathbb{N}^{*}\right\}\right.
$$

This is a simple optimization problem that does not require the use of optimization algorithm; the exhaustive (or brute-force) search is easily and quickly achievable (in practice $N \in$ [1:100]

\subsection{Theoretical validation of the stochastic persistence}

In order to understand the stochastic persistence interest, it is necessary to remind the MSE decomposition (variance and bias [47]) as described below:

$$
M S E=\operatorname{Var}(\widehat{G H I}(t+h))+(\operatorname{Bias}(\widehat{G H I}(t+h), G H I(t+h)))^{2}
$$

With $\operatorname{Var}(\widehat{G H I}(t+h))=\mathbb{E}\left[(\widehat{G H I}(t+h)-\mathbb{E}[\widehat{G H I} I(t+h)])^{2}\right]$

And $(\operatorname{Bias}(\widehat{G H I}(t+h), G H I(t+h)))^{2}=\mathbb{E}\left[(\mathbb{E}[\widehat{G H} I(t+h)]-G H I(t+h))^{2}\right]$ Equation 15

Whether one uses the smart persistence or one of the two stochastic persistences described previously, the biases are similar; indeed, $\mathbb{E}[\widehat{G H} I(t+h)]$ is identical in the three cases, only the variance changes and is directly impacted by the mean of CSI or $\Delta$. In conclusion, the variance part and so MSE decreases with the stochastic persistence use. A validation can be operated considering two components of the measured GHI signal (mean and noise). Note that here the determinist part $(T)$ is not the $C S$ described previously but only an average value (or trend) and the noise a random variable ( $\varepsilon$. So with the condition $T(t)+\varepsilon(t)<C S(t)$, we obtain:

$G H I(t)=T(t)+\varepsilon(t)$ with $t \in \mathbb{N}^{*}, T \in \mathcal{R}^{+}$and $\varepsilon \in \mathcal{R}$

Equation 16

In the additive case, $\mathbb{E}[\varepsilon(t)]=0 \forall t$ according to the white noise definition around a signal. Moreover with the stochastic persistence, there will be a persistence of the trend but not of the noise, so: $\lim _{N \rightarrow \infty} \hat{\varepsilon}(t+h)=0 \forall t, h$. For the smart persistence $(S P)$ and the stochastic persistences $\left(S t P^{+}\right.$and $\left.S t P^{\mathrm{x}}\right)$, we obtain three important results:

Result 1: $\operatorname{Var}_{S t P}(\widehat{G H} I(t+h))<\operatorname{Var}_{S P}(\widehat{G H I}(t+h))$ Equation 17

Result 2 : $\operatorname{Bias}_{S P}(\widehat{G H I}(t+h), G H I(t+h))=\operatorname{Bias}_{S t P}(\widehat{G H} I(t+h), G H I(t+h)) \quad$ Equation 18

Result 3 : $n R M S E_{S t P}(\widehat{G H I}(t+h))<n R M S E_{S P}(\widehat{G H I}(t+h))$ Equation 20 
The proofs of these three results are available in the annex of this paper. The most important result (the third) induces that the stochastic persistence improves (at least in theory) the prediction done with the smart persistence or the simple persistence.

\section{Results}

The forecasting of GHI needs usually a cleaning and a preparation of the dataset. Mistakes often appear in the temporal series of solar data due to problems with the acquisition system; an automatic quality check used in the frame of GEOSS project (Group on Earth Observation System of System) [48] has been applied to the data. The process to estimate the quality of the data [49] and the procedure applied to flag suspicious or erroneous measurements is described in detail in [50]. Then, we applied a filter on the datasets which remove all the data that correspond to a solar elevation angle lower than $10^{\circ}$, in order to removing the night hours [19]. In this section, we will compare the prediction results of stochastic persistence with those related to two well know machine learning tools: ARMA (more precisely $A R$ with $M A$ part) and $M L P$. The training and optimization phases of these models are classic and interesting reader could find all the methodologies of prediction in [14,19]. In order to objectively compare the results, we propose the k-fold sampling use [51], the dataset is divided in ten samples (each with $80 \%$ of the total available data) and every sample is used at least one time for the training (only for the machine learning tools and not for the different persistences) and one time for the test ( $20 \%$ of the data and use for all models). This method induces to avoid the problems which can results of measurements of the dataset. The datasets used in this study are time series of measurements of global horizontal solar irradiation $(G H I)$ in two different sites with different meteorological situations. The first dataset is provided by the station of Ajaccio from 1998 to 2009 (Corsica, France, $41^{\circ} 55$ N, $8^{\circ} 44 \mathrm{E}, 4 \mathrm{~m}$ asl), it is located near the Mediterranean Sea $(100 \mathrm{~m})$ and nearby mountains (1000 m altitude at $40 \mathrm{~km}$ from the site). This specific geographical configuration and the island context make the nebulosity difficult to forecast. The Mediterranean climate is characterized by hot summers with abundant sunshine and mild, dry and clear wintersand. The second one is constituted by measurements in Tilos from 2015 to 2016 (Tilos Island, Greece, $36^{\circ} 24 \mathrm{~N}, 27^{\circ} 22 \mathrm{E}, 96 \mathrm{~m}$ asl), which is a small island in the Dodecanese archipelago, the tallest mountain is about $650 \mathrm{~m}$ high and the cloud occurrences are much less frequent than in Ajaccio. These stations are equipped with pyranometers (CM 11 Kipp \& Zonen) and standard meteorological sensors (pressure, temperature, etc.), the solar data are measured and stored with a time step equal to $1 \mathrm{~min}$.

\subsection{Hourly time granularity in Ajaccio}


In this subsection, the data of Ajaccio are used. The tested horizons are from 1 hour to 6 hours. Before to expose the results of prediction, we propose to develop the optimization step of the $S t P^{+}$and $S t P^{\mathrm{x}}$ formalisms. In Figs 3 and 4 are represented the prediction errors in term of size of sliding windows. The optimization concerns the $N$ parameter described in Eq 12. The optimized models are related to $N$ giving the lowest value of $n R M S E$. For example, in the first figure and concerning the horizon 1 hour, the $S t P^{\mathrm{x}}$ constructed with $N=1$, gives the best performance. In this case, the $S t P^{\mathrm{x}}$ is equivalent to classical $S P$ estimator (see Eq 11). We remark that the higher the horizon is, the higher the optimized $N$ value is in the two cases $S t P^{\mathrm{x}}$ and $S t P^{+}$, but also that a known conclusion is verified: the prediction error increases with the horizon. The additive mode is less reliable than the multiplicative mode with the hourly time granularity. This kind of stochastic time series seems follow a multiplicative scheme.

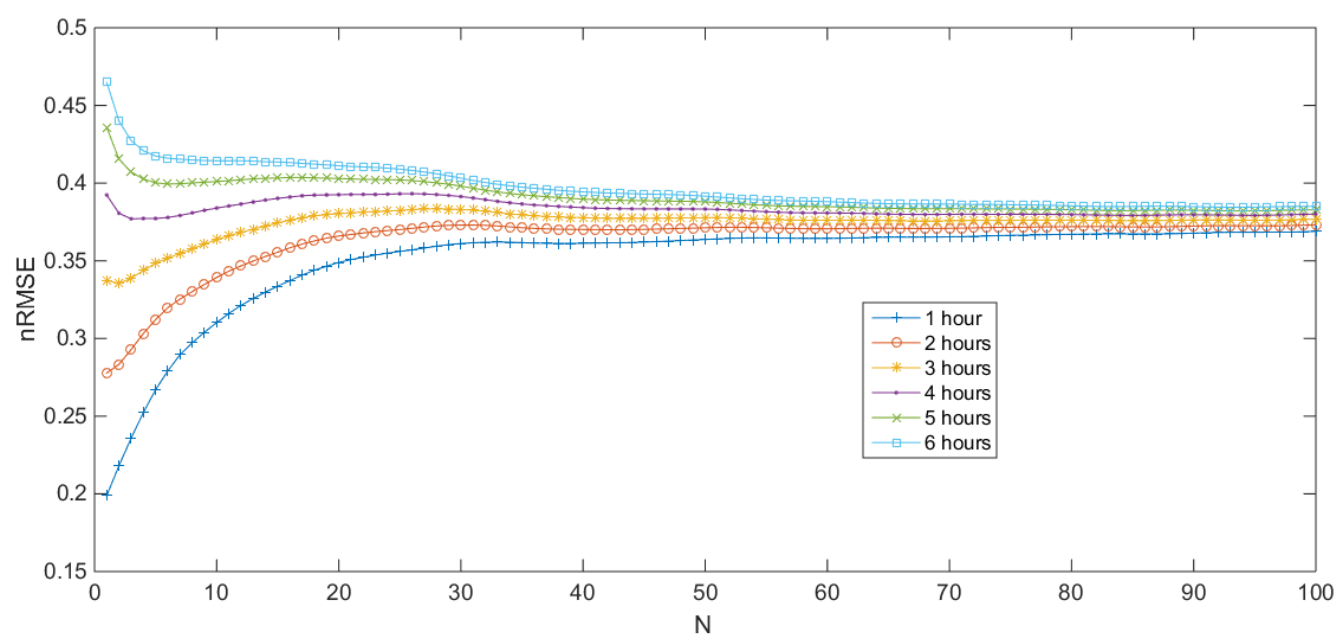

Figure 3. Prediction error (from horizon 1 hours to 6 hours in Ajaccio) in term of size of the sliding window concerning the $S t P^{\mathrm{x}}$ (Eq. 12)

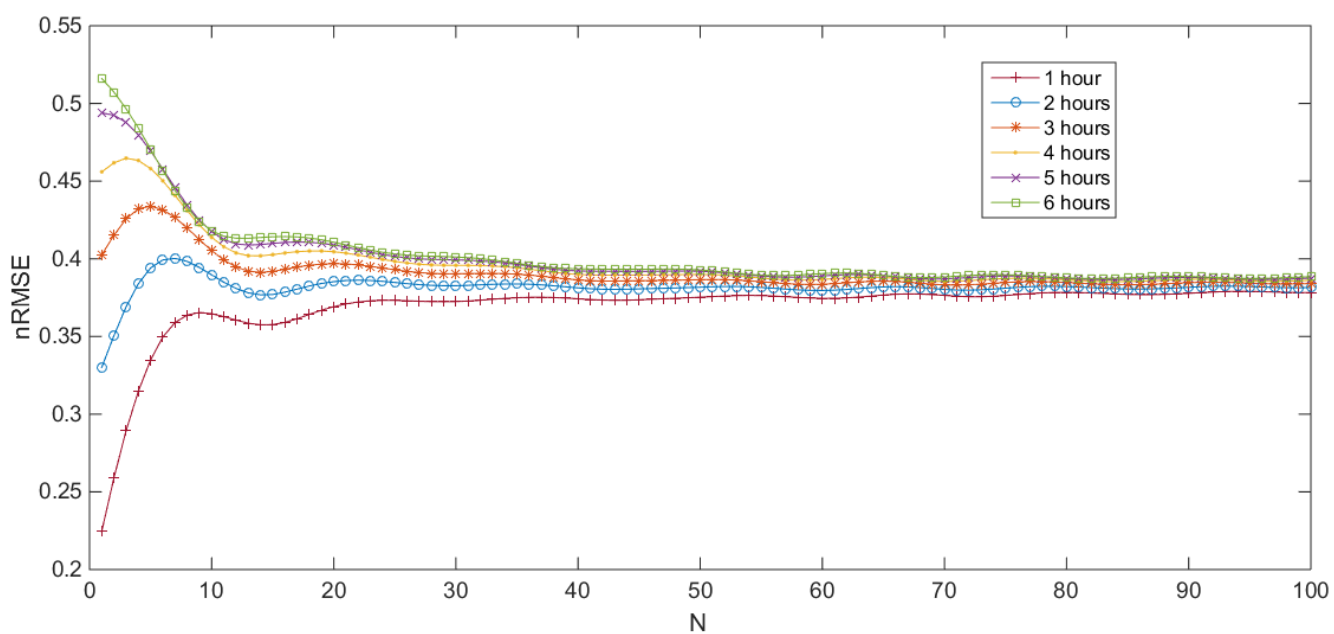

Figure 4. Prediction error (from horizon 1 hour to 6 hour in Ajaccio) in term of size of the sliding window concerning the $S t P^{+}$(Eq. 12) 
The best configurations for each horizon are given in the Table 2 .

\begin{tabular}{|c|c|c|c|c|c|c|}
\hline & 1 hour & 2 hours & 3 hours & 4 hours & 5 hours & 6 hours \\
\hline St $^{+}$ & 1 & 1 & 71 & 84 & 83 & 82 \\
\hline StP $^{\mathbf{x}}$ & 1 & 1 & 2 & 3 & 94 & 93 \\
\hline
\end{tabular}

Table 2. Value of the optimized $N$ for each horizon (Ajaccio)

345 Now the stochastic persistences are optimized, it is essential to compare the results of prediction with classical methodologies $(S P, A R$ and $M L P)$. Fig 5 shows the errors related to all these models.

347

348

349 If the $A R$ and $M L P$ models give the best results, using $S t P^{x}$ allows to improve the prediction related to

$350 S P$. The numerical values of $n R M S E$ are given is Table 3. We note that $S t P^{+}$and mainly $S t P^{\mathrm{x}}$ represent 351 a high improvements compared to $S P$ and $P$ for long horizons (from $\mathrm{h}+4$ to $\mathrm{h}+6$ ) and reach a good 352 level of accuracy compared with $A R$ and $M L P$.

Figure 5. $n R M S E$ Vs horizon for the 5 studied models (Ajaccio)

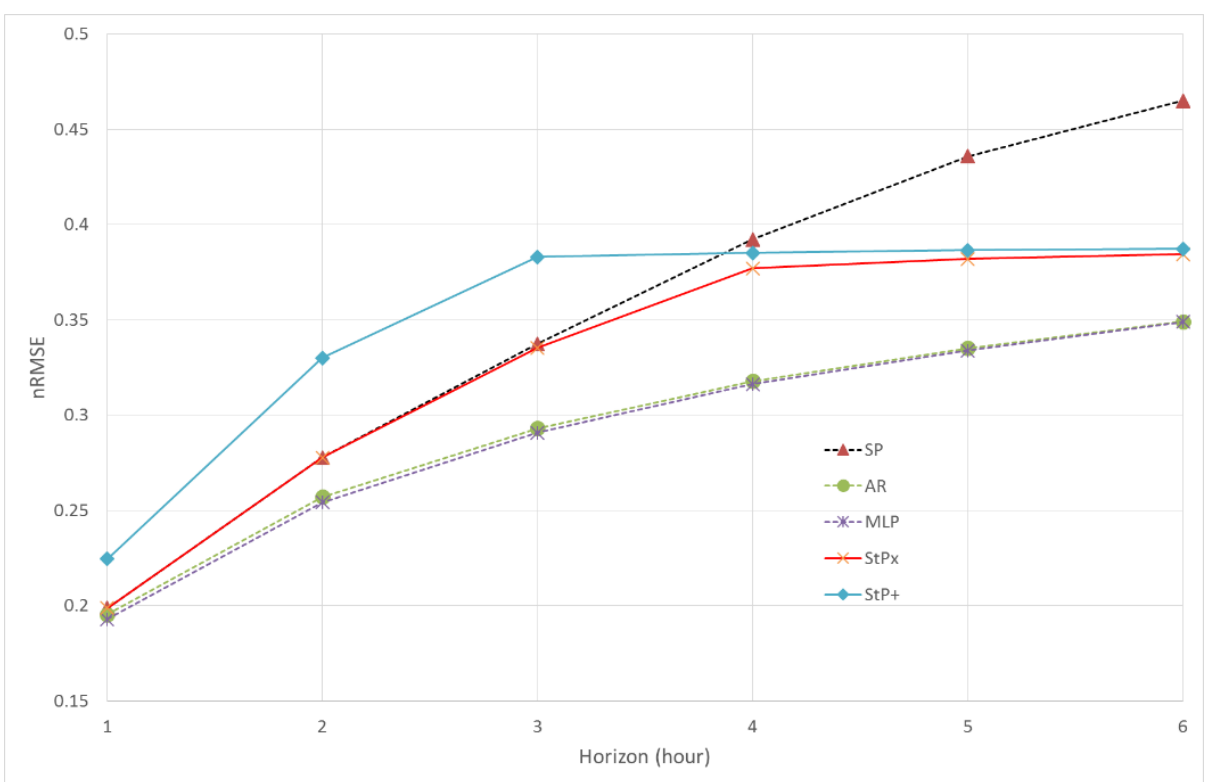

\begin{tabular}{lcccccc} 
horizons & $\boldsymbol{P}$ & $\boldsymbol{S P}$ & $\boldsymbol{A R}$ & $\mathbf{M L P}$ & St $^{+}$ & St $^{\boldsymbol{x}}$ \\
\hline 1 hour & 0.3442 & 0.1988 & 0.1954 & 0.1929 & 0.2246 & 0.1988 \\
\hline 2 hours & 0.5981 & 0.2778 & 0.2570 & 0.2543 & 0.3301 & 0.2778 \\
\hline 3 hours & 0.8061 & 0.3375 & 0.2931 & 0.2908 & 0.3830 & 0.3353 \\
\hline 4 hours & 0.9662 & 0.3923 & 0.3179 & 0.3163 & 0.3852 & 0.3770 \\
\hline 5 hours & 1.0741 & 0.4359 & 0.3352 & 0.3340 & 0.3865 & 0.3820 \\
\hline 6 hours & 1.1269 & 0.4650 & 0.3492 & 0.3490 & 0.3873 & 0.3844 \\
& \multicolumn{6}{c}{ Table 3. $n R M S E$ for all models (Ajaccio) } \\
\end{tabular}


It is now interesting to show profiles of prediction in order to visually verify the quality of the 355 stochastic persistence. In Figure 6 is shown the 1 hour horizon case concerning $S t P^{+}$and $S t P^{\mathrm{x}}$.

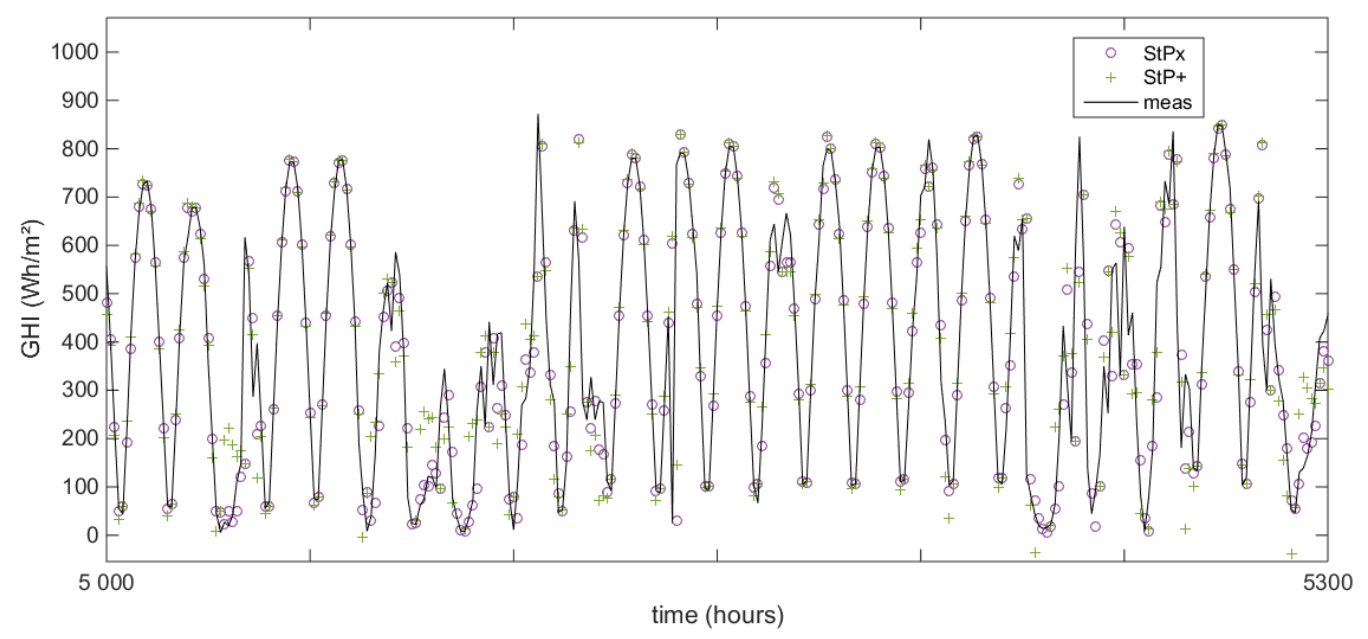

357 Figure 6. Profile of prediction related to $S t P^{+}$and $S t P^{\mathrm{x}}$ between the $5000^{\text {th }}$ and $5300^{\text {th }}$ hour (spring in Ajaccio)

359 We see a very good accordance between predictions and measures even when the variability is important. In the next subsection, we will verify the conclusion drawn here with another kind of time granularity and another location.

As for Ajaccio using hourly data, for Tilos with 15 min data, the first step is to optimize the stochastic persistences using Figs 7 and 8 . 


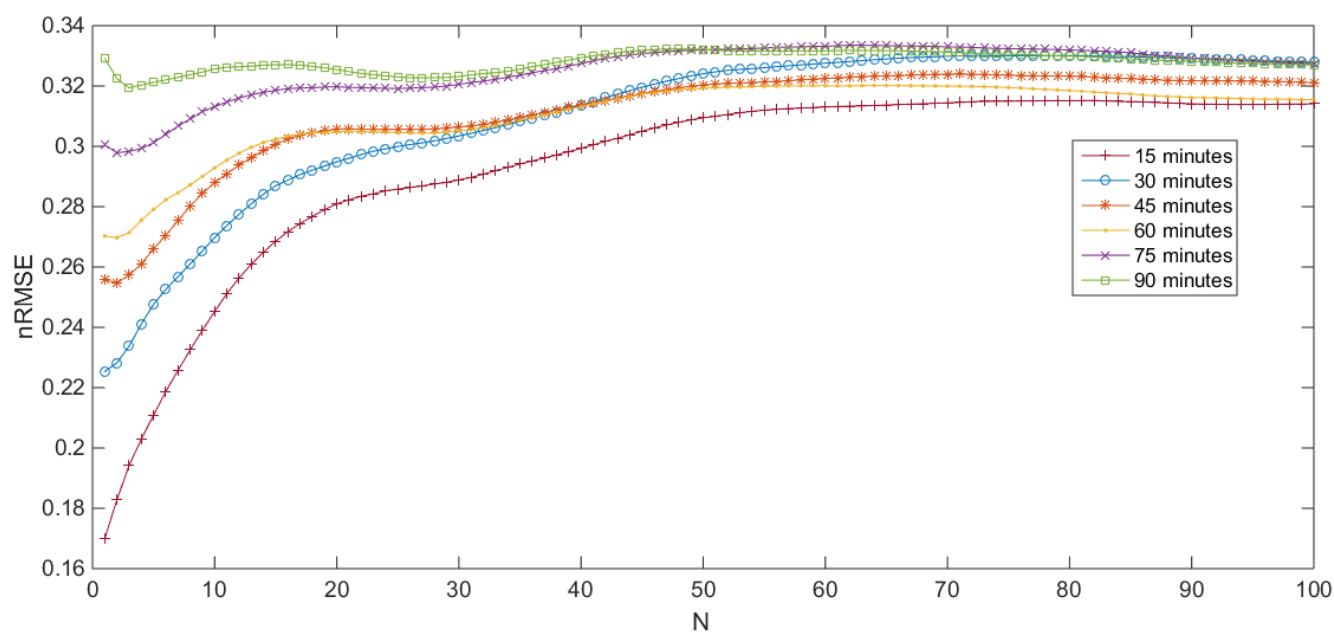

Figure 7. Prediction error (from horizon 15 minutes to 90 minutes in Tilos) in term of size of the sliding window concerning the $S t P^{\mathrm{x}}$ (Eq 12)

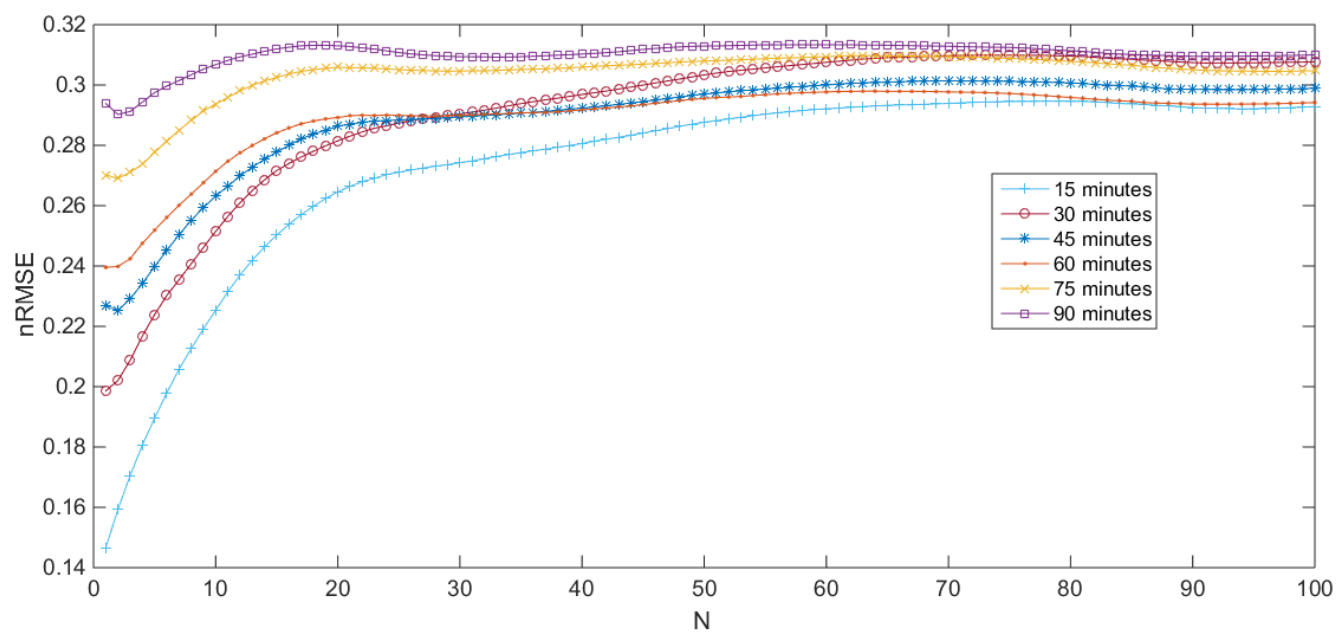

Figure 8. Prediction error (from horizon 15 minutes to 90 minutes in Tilos) in term of size of the sliding window concerning the $S t P^{+}$(Eq 12)

The conclusions are similar to the hourly case for Ajaccio, but here the additive mode seems more relevant than the multiplicative mode. In fact, we think this phenomenon is related to the clear sky estimation. In hourly case, it is less important to have a precise $C S$ function (smoothing related to the hourly aggregation). The hourly sum tends to minimize the impact of the quality of the CS modeling. For lower time granularity, the consequence of using a good clear sky model becomes very important with the use of multiplicative mode and the division by $C S$ (ratio to trend). Indeed, introducing briefly the condition number of a problem as tool measuring how the output value of the modeling can change for a small change in the input argument, we can certainly consider that our CS estimation is not efficient for the concerning problem. It is really difficult to improve the $C S$ modeling because a lot of parameters (not always available) change during the year, the day and each hour. So as minimal conclusion, we can consider that the additive scheme is the most interesting when the time granularity 
decreases. Concerning the multiplicative case, the $C S$ estimation induces an ill-conditioned problem not really performant. In Table 4 are listed the optimized value of $N$ parameters.

\begin{tabular}{|c|c|c|c|c|c|c|}
\hline & $15 \mathrm{~min}$ & $30 \mathrm{~min}$ & $45 \mathrm{~min}$ & $60 \mathrm{~min}$ & $75 \mathrm{~min}$ & $90 \mathrm{~min}$ \\
\hline$S t P^{+}$ & 1 & 1 & 2 & 2 & 2 & 3 \\
\hline$S t P^{\mathrm{x}}$ & 1 & 1 & 2 & 1 & 2 & 2 \\
\hline
\end{tabular}

Now the stochastic persistences are optimized, we can compare the prediction errors with the other reference models (SP, AR and MLP). Figure 9 shows this comparison concerning 6 horizons from 15 minutes to 90 minutes.

392

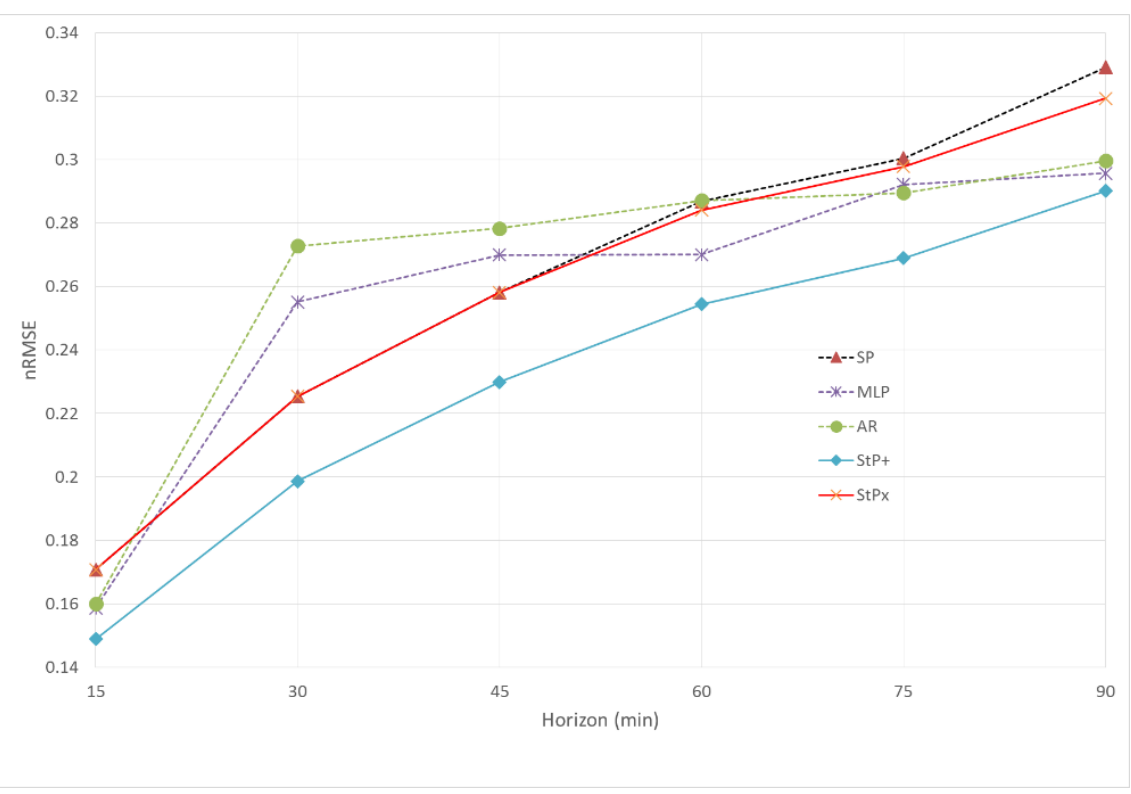

Figure 9. $n R M S E$ vs horizon for the 5 studied models (Tilos)

Bellow 60 minutes, the three persistences $\left(S P, S t P^{\mathrm{x}}\right.$ and $\left.S t P^{+}\right)$give better results than sophisticated methods. The best model is, for all the horizon, StP $P^{+}$. The $n R M S E$ values are given in Table 5 .

\begin{tabular}{|c|c|c|c|c|c|c|}
\hline horizons & $P$ & $S P$ & $A R$ & $M L P$ & $S t P^{+}$ & $S t P^{x}$ \\
\hline $15 \mathrm{~min}$ & 0.1929 & 0.1708 & 0.1600 & 0.1587 & 0.1489 & 0.1708 \\
\hline $30 \mathrm{~min}$ & 0.2804 & 0.2254 & 0.2728 & 0.2552 & 0.1986 & 0.2254 \\
\hline $45 \mathrm{~min}$ & 0.3419 & 0.2582 & 0.2784 & 0.2695 & 0.2299 & 0.2582 \\
\hline $60 \mathrm{~min}$ & 0.3988 & 0.2869 & 0.2872 & 0.2701 & 0.2545 & 0.2841 \\
\hline $75 \mathrm{~min}$ & 0.4528 & 0.3005 & 0.2896 & 0.2922 & 0.269 & 0.2979 \\
\hline $90 \mathrm{~min}$ & 0.5013 & 0.3291 & 0.2997 & 0.2957 & 0.2902 & 0.3194 \\
\hline
\end{tabular}

Table 5. nRMSE for all models (Tilos) 
We observe that the prediction is more reliable in the 15 minutes case than in the hourly case. Probably because there are some weather afterglow for very short duration. For this time granularity, the stochastic persistence model is the best whatever the time horizon is.

As for the hourly case in Ajaccio, in Fig 10, is presented a comparison between measured and forecasted values on a period of several days for $S t P^{+}$and $S t P^{\mathrm{x}}$ (15 minutes ahead).

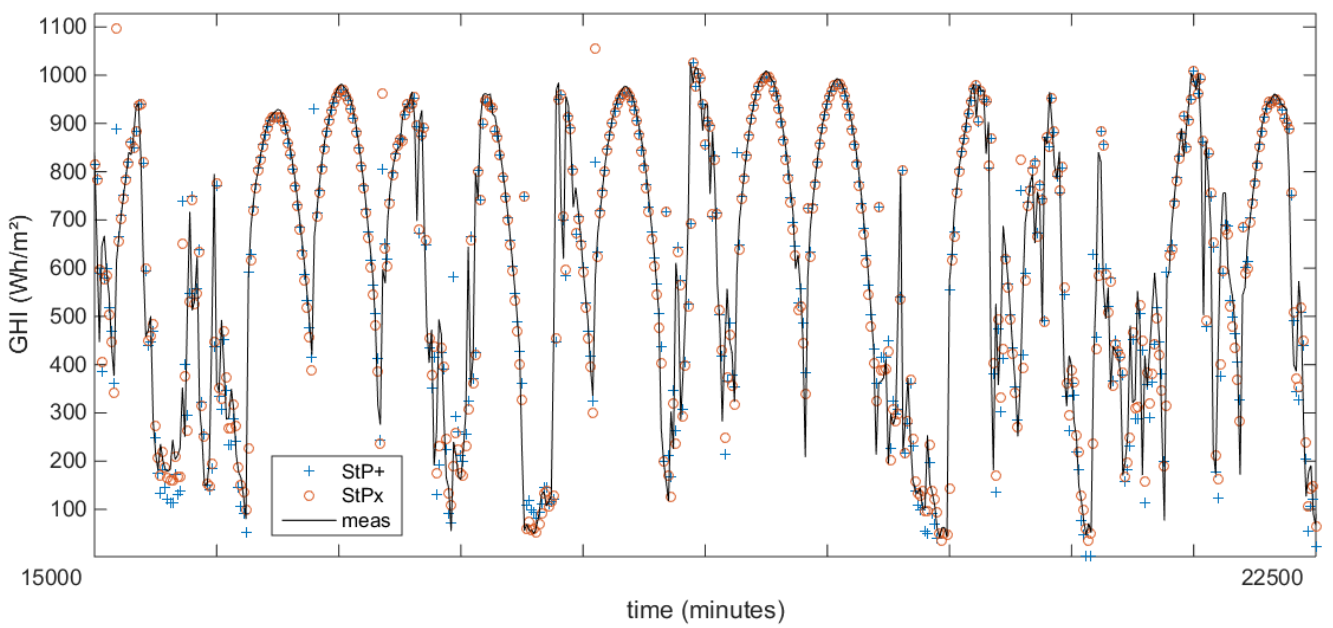

Figure 10. Profile of prediction related to $S t P^{+}$and $S t P^{\mathrm{x}}$ between the $15000^{\text {th }}$ and $22500^{\text {th }}$ minutes (spring in Tilos)

We observe a high accordance between measurements and predictions and that predicted values of $G H I$ by $S t P^{+}$are better than ones predicted by $S t P^{x}$.

\section{Conclusion}

A new forecasting methodology was presented, it is based on the assumption that GHI signal has two components: a stochastic and a deterministic parts. Two stochastic methods were developed an additive and a multiplicative schemes. The stochastic persistence allows to easily establish GHI prediction with a good accuracy without the need of large historical data collection.

The stochastic persistence was experimentally tested in two sites Ajaccio, Corsica, France and Tilos, Greece with two time granularities (1 hour and $15 \mathrm{~min}$ ). It appeared that the results obtained by stochastic persistence model are systematically better than those obtained with classical or smart persistences; For 1 hour horizon, they are relatively close to those obtained with some sophisticated machine learning tools. For other prediction horizons and time granularities (15 minutes), the data driven methods are less interesting than the stochastic persistence in the additive mode. 
421 In the hourly case, the stochastic persistence should be considered as naïve predictor in order to

422 compare and valid more sophisticated methods of machine learning. Moreover, the reliability of the 423 multiplicative stochastic persistence method, is not so very far from those obtained by sophisticated 424 methods.

425 It would probably be interesting to construct error metric related to this tool, especially a new version 426 of the skill score which is actually the most common parameter in the production of global radiation.

427 For the 15 minutes case, the stochastic persistence gives very good results mainly with the additive 428 scheme. Some investigation related to clear sky modeling concerning the very short time granularity 429 should be undertaken with the goal to improve the multiplicative scheme of the stochastic persistence 430 and to valid the conclusions drawn here.

431 Thus the developed forecasted tool (with its two versions, additive and multiplicative) showed very 432 good performances for a forecasting method that does not need a long and rare set of historical data 433 and complicated training phase for nowcasting purpose. 
435

436

Result 1: $\operatorname{Var}_{S t P}(\widehat{G H} I(t+h))<\operatorname{Var}_{S P}(\widehat{G H} I(t+h))$

437

Result 2: $\operatorname{Bias}_{S P}(\widehat{G H} I(t+h), G H I(t+h))=\operatorname{Bias}_{S t P}(\widehat{G H I}(t+h), G H I(t+h))$

438

Result 3 : $n R M S E_{S t P}(\widehat{G H I}(t+h))<n R M S E_{S P}(\widehat{G H I}(t+h))$

439

$\underline{\text { Proof of results } 1}$

440

We have:

441

$\operatorname{Var}_{S P}(\widehat{G H} I(t+h))=\operatorname{Var}_{S P}(\widehat{T}(t+h)+\hat{\varepsilon}(t+h))$

442

Related to the variance definition, we can write:

443

$\operatorname{Var}_{S P}(\widehat{G H I}(t+h))=\mathbb{E}\left[(\widehat{T}(t+h)+\hat{\varepsilon}(t+h)-\mathbb{E}[\widehat{T}(t+h)+\hat{\varepsilon}(t+h)])^{2}\right]$

444

If $\hat{\varepsilon}(t+h)$ is a white noise, so:

445

$\operatorname{Var}_{S P}(\widehat{G H I}(t+h))=\mathbb{E}\left[\left(\widehat{T}(t+h)+\hat{\varepsilon}(t+h)-\mathbb{E}[\widehat{T}(t+h))^{2}\right]\right.$

446

Signifying that $\quad \operatorname{Var}_{S t P}(\widehat{G H I}(t+h))=\mathbb{E}\left[(\widehat{T}(t+h)-\mathbb{E}[\widehat{T}(t+h)])^{2}\right]<\operatorname{Var}_{S P}(\widehat{G H I}(t+h))$

447

448

$\underline{\text { Proof of results } 2}$

449 Related to the bias definition, we have:

$$
\left(\operatorname{Bias}_{S P}(\widehat{G H I}(t+h), G H I(t+h))\right)^{2}=\mathbb{E}\left[(\mathbb{E}[\widehat{T}(t+h)+\hat{\varepsilon}(t+h)]-T(t+h)+\varepsilon(t+h))^{2}\right]
$$

450 If $\hat{\varepsilon}(t+h)$ is a write noise, we also can consider:

451

$$
\left(\operatorname{Bias}_{S P}(\widehat{G H I}(t+h), G H I(t+h))\right)^{2}=\mathbb{E}\left[(\mathbb{E}[\widehat{T}(t+h)]-T(t+h)+\varepsilon(t+h))^{2}\right]
$$

452

453 So, we can write that:

454

$$
\left(\operatorname{Bias}_{S t P}(\widehat{G H I}(t+h), G H I(t+h))\right)^{2}=\left(\operatorname{Bias}_{S P}(\widehat{G H I}(t+h), G H I(t+h))\right)^{2}
$$


456 The results 1 and 2 lead to $M S E_{S t P}(\widehat{G H} I(t+h))<M S E_{S P}(\widehat{G H} I(t+h))$, according to the definition 457 of the normalized root mean square error (nRMSE, []), we obtain:

$458 \quad n R M S E_{S t P}(\widehat{G H} I(t+h))<n R M S E_{\mathrm{SP}}(\widehat{G H I}(t+h))$ 

Systems Operation. London: Springer London; 2013.

464

[2] Azofra D, Martínez E, Jiménez E, Blanco J, Azofra F, Saenz-Díez JC. Comparison of the influence of photovoltaic and wind power on the Spanish electricity prices by means of artificial intelligence techinques. Renew Sustain Energy Rev 2015;42:532-42. doi:10.1016/j.rser.2014.10.048.

[3] Polo J, Gastón M, Vindel JM, Pagola I. Spatial variability and clustering of global solar irradiation in Vietnam from sunshine duration measurements. Renew Sustain Energy Rev 2015;42:1326-34. doi:10.1016/j.rser.2014.11.014.

[4] Hoff TE, Perez R. Modeling PV fleet output variability. Sol Energy 2012;86:2177-89. doi:10.1016/j.solener.2011.11.005.

[5] Badescu V. Modeling solar radiation at the earth's surface: recent advances. Springer; 2008.

[6] Perez R, Kivalov S, Schlemmer J, Hemker Jr. K, Hoff TE. Short-term irradiance variability: Preliminary estimation of station pair correlation as a function of distance. Sol Energy 2012;86:2170 6. doi:10.1016/j.solener.2012.02.027.

[7] De Felice M, Petitta M, Ruti PM. Short-term predictability of photovoltaic production over Italy. Renew Energy 2015;80:197-204. doi:10.1016/j.renene.2015.02.010.

[8] Cros S, Buessler E, Huet L, Sébastien N, Schmutz N. The benefits of intraday solar irradiance forecasting to adjust the day-ahead scheduled PV power. 2015.

[9] Sylvain Cros OL. Clear sky models assessment for an operational PV production forecasting solution 2013.

[10] Almeida MP, Perpiñán O, Narvarte L. PV power forecast using a nonparametric PV model. Sol Energy 2015;115:354-68. doi:10.1016/j.solener.2015.03.006.

[11] Diagne HM, Lauret P, David M. Solar irradiation forecasting: state-of-the-art and proposition for future developments for small-scale insular grids, n.d.

[12] Paulescu M, Mares O, Eugenia P, Stefu N, Pacurar A, Calinoiu D, et al. Nowcasting solar irradiance using the sunshine number. Energy Convers Manag 2014;79:690-7. doi:10.1016/j.enconman.2013.12.048. 
[13] De Gooijer JG, Hyndman RJ. 25 years of time series forecasting. Int J Forecast 2006;22:44373. doi:10.1016/j.ijforecast.2006.01.001.

[14] Voyant C, Motte F, Fouilloy A, Notton G, Paoli C, Nivet M-L. Forecasting method for global radiation time series without training phase: comparison with other well-known prediction methodologies. Energy 2017;120:199-208.

[15] Join C, Voyant C, Fliess M, Muselli M, Nivet ML, Paoli C, et al. Short-term solar irradiance and irradiation forecasts via different time series techniques: A preliminary study, 2014.

[16] Hocaoglu FO. Stochastic approach for daily solar radiation modeling. Sol Energy 2011;85:278-87. doi:10.1016/j.solener.2010.12.003.

[17] Kristensen. Parameter estimation in stochastic grey-box models. Automatica 2004.

[18] Mustacchi C, Cena V, Rocchi M. Stochastic simulation of hourly global radiation sequences. Sol Energy 1979;23:47-51. doi:10.1016/0038-092X(79)90042-2.

[19] Lauret P, Voyant C, Soubdhan T, David M, Poggi P. A benchmarking of machine learning techniques for solar radiation forecasting in an insular context. Sol Energy 2015;112:446-57. doi:10.1016/j.solener.2014.12.014.

[20] Pedro HTC, Coimbra CFM. Nearest-neighbor methodology for prediction of intra-hour global horizontal and direct normal irradiances. Renew Energy 2015;80:770-82. doi:10.1016/j.renene.2015.02.061.

[21] Kaplanis SN. New methodologies to estimate the hourly global solar radiation; Comparisons with existing models. Renew Energy 2006;31:781-90. doi:10.1016/j.renene.2005.04.011.

[22] Perez R, Hoff T, Dise J, Chalmers D, Kivalov S. The Cost of Mitigating Short-term PV Output Variability. Energy Procedia 2014;57:755-62. doi:10.1016/j.egypro.2014.10.283.

[23] Domingos P. The Role of Occam's Razor in Knowledge Discovery. Data Min Knowl Discov 1999;3:409-25. doi:10.1023/A:1009868929893.

[24] Divya U, Pasupathi C. Survey on Machine Learning Approaches for Solar Irradiation Prediction. Int J Eng Sci Res Technol 2014;3:478-82.

[25] Perera KS, Aung Z, Woon WL. Machine Learning Techniques for Supporting Renewable Energy Generation and Integration: A Survey. Data Anal. Renew. Energy Integr., Springer; 2014, p. 81-96.

\footnotetext{
[26] Bayesian Learning for Neural Networks | Radford M. Neal | Springer. n.d.
} 

methods for solar radiation forecasting: A review. Renew Energy 2017;105:569-82.

[28] Mellit A, Kalogirou SA, Hontoria L, Shaari S. Artificial intelligence techniques for sizing

522 photovoltaic systems: A review. Renew Sustain Energy Rev 2009;13:406-19. 523 doi:10.1016/j.rser.2008.01.006.

524 [29] Voyant C, Paoli C, Muselli M, Nivet M-L. Multi-horizon solar radiation forecasting for 525 Mediterranean locations using time series models. Renew Sustain Energy Rev 2013;28:44-52.

526 [30] Quora. Is Machine Learning Overhyped? Forbes n.d.

527 https://www.forbes.com/sites/quora/2016/07/18/is-machine-learning-overhyped/ (accessed September $52827,2017)$.

529 [31] Kim TY, Oh KJ, Kim C, Do JD. Artificial neural networks for non-stationary time series. 530 Neurocomputing 2004;61:439-47. doi:10.1016/j.neucom.2004.04.002.

531 [32] Marty C, Philipona R. Clear-sky index to separate clear-sky from cloudy-sky situations in 532 climate research. Geophys Res Lett 2000;27:2649-2652.

[33] Antonanzas J, Osorio N, Escobar R, Urraca R, Ascacibar FJ, Antonanzas F. Review of photovoltaic power forecasting. Sol Energy 2016;136. doi:10.1016/j.solener.2016.06.069.

[34] Ahmad A, Anderson TN, Lie TT. Hourly global solar irradiation forecasting for New Zealand. Sol Energy 2015;122:1398-408. doi:10.1016/j.solener.2015.10.055.

[35] Sanfilippo A, Martín Pomares L, Mohandes N, Perez-Astudillo D, Bachour D. An adaptive multi-modeling approach to solar nowcasting. Sol Energy 2016;125:77-85. doi:10.1016/j.solener.2015.11.041.

[36] Martín Pomares L, Zarzalejo L, Polo J, Navarro A, Marchante R, Cony M. Prediction of global solar irradiance based on time series analysis: Application to solar thermal power plants energy production planning. Sol Energy 2010;84:1772-81. doi:10.1016/j.solener.2010.07.002.

[37] Wang Y, wu L. On Practical Challenges of Decomposition-Based Hybrid Forecasting Algorithms for Wind Speed and Solar Irradiation,. Energy 2016;112. doi:10.1016/j.energy.2016.06.075.

[38] Voyant C, Haurant P, Muselli M, Paoli C, Nivet M-L. Time series modeling and large scale global solar radiation forecasting from geostationary satellites data. Sol Energy 2014;102:131-42. 
548 [39] Chu Y, Coimbra CFM. Short-term probabilistic forecasts for Direct Normal Irradiance. Renew 549 Energy 2017;101:526-36.

550 [40] Chu Y, Li M, T.C. Pedro H, F.M. Coimbra C. Real-time prediction intervals for intra-hour 551 DNI forecasts. Renew Energy 2015;83. doi:10.1016/j.renene.2015.04.022.

552 [41] Urraca R, Antonanzas J, Alía Martínez M, Ascacibar FJ, Antonanzas F. Smart baseline models 553 for solar irradiation forecasting. Energy Convers Manag 2016;108:539-48. 554 doi:10.1016/j.enconman.2015.11.033.

555 [42] Brown CE. Coefficient of Variation. Appl. Multivar. Stat. Geohydrology Relat. Sci., Springer, 556 Berlin, Heidelberg; 1998, p. 155-7. doi:10.1007/978-3-642-80328-4_13.

557 [43] Ineichen P. A broadband simplified version of the Solis clear sky model. Sol Energy $558 \quad 2008 ; 82: 758-62$. doi:10.1016/j.solener.2008.02.009.

559 [44] Mueller RW, Dagestad KF, Ineichen P, Schroedter-Homscheidt M, Cros S, Dumortier D, et al. 560 Rethinking satellite-based solar irradiance modelling: The SOLIS clear-sky module. Remote Sens 561 Environ 2004;91:160-74. doi:10.1016/j.rse.2004.02.009.

562 [45] Masani P. Shift invariant spaces and prediction theory. Acta Math 1962;107:275-90. 563 doi:10.1007/BF02545791.

564 [46] Cartier P, Perrin Y. Integration over finite sets. Nonstandard Anal. Pract., Springer, Berlin, 565 Heidelberg; 1995, p. 185-204. doi:10.1007/978-3-642-57758-1_9.

566 [47] Neville J, Jensen D. A bias/variance decomposition for models using collective inference. 567 Mach Learn 2008;73:87-106. doi:10.1007/s10994-008-5066-6.

568 [48] GEOSS n.d. https://www.earthobservations.org/geoss.php (accessed July 26, 2017).

569 [49] Korany M, Boraiy M, Eissa Y, Aoun Y, Abdel Wahab MM, Alfaro SC, et al. A database of 570 multi-year (2004-2010) quality-assured surface solar hourly irradiation measurements for the 571 Egyptian territory. Earth Syst Sci Data 2016;8:105-113.

572 [50] David M, Ramahatana F, Trombe P-J, Lauret P. Probabilistic forecasting of the solar 573 irradiance with recursive ARMA and GARCH models. Sol Energy 2016;133:55-72.

574 [51] Wiens TS, Dale BC, Boyce MS, Kershaw GP. Three way k-fold cross-validation of resource 575 selection functions. Ecol Model 2008;212:244-55. doi:10.1016/j.ecolmodel.2007.10.005. 
578

579 
606

607

\section{List of Tables}

Table 1 . Short summary concerning the persistence models.

Table 2. Value of the optimized $N$ for each horizon (Ajaccio)

Table 3. $n R M S E$ for all models (Ajaccio)

Table 4. Value of the optimized $N$ for each horizon (Tilos)

Table 5. nRMSE for all models (Tilos)

\section{List of figures}

Figure 1. Principle of the stochastic persistence based on an additive scheme

Figure 2. Principle of the stochastic persistence based on a multiplicative scheme

Figure 3. Prediction error (from horizon 1 hours to 6 hours in Ajaccio) in term of size of the sliding window concerning the $S t P^{\mathrm{x}}$ (Eq. 12)

Figure 4. Prediction error (from horizon 1 hour to 6 hour in Ajaccio) in term of size of the sliding window concerning the $\operatorname{StP}^{+}$(Eq. 12)

Figure 5. nRMSE Vs horizon for the 5 studied models (Ajaccio)

Figure 6. Profile of prediction related to $S t P^{+}$and $S t P^{\mathrm{x}}$ between the $5000^{\text {th }}$ and $5300^{\text {th }}$ hour (spring in Ajaccio)

Figure 7. Prediction error (from horizon 15 minutes to 90 minutes in Tilos) in term of size of the sliding window concerning the $S t P^{\mathrm{x}}(\mathrm{Eq} 12)$

Figure 8. Prediction error (from horizon 15 minutes to 90 minutes in Tilos) in term of size of the sliding window concerning the $\operatorname{StP}^{+}$(Eq 12)

Figure 9. $n R M S E$ vs horizon for the 5 studied models (Tilos)

Figure 10. Profile of prediction related to $S t P^{+}$and $S t P^{\mathrm{x}}$ between the $15000^{\text {th }}$ and $22500^{\text {th }}$ minutes (spring in Tilos) 